\title{
Monocyte-derived Wnt5a regulates inflammatory lymphangiogenesis
}

Cell Research (2016) 26:262-265. doi:10.1038/cr.2015.105; published online 4 September 2015

\section{Dear Editor,}

Lymphatic research signifies a field of explosive discovery in recent years [1-3]. The lymphatic network penetrates most tissues in the body and its dysfunction has been found in a broad spectrum of disorders from inflammation to cancer metastasis and transplant rejection. However, to date, there are few effective treatments for lymphatic diseases. It is therefore important and urgent to investigate the fundamental mechanisms of pathological lymphangiogenesis (LG, the formation of new lymphatic vessels) for the development of new therapeutic strategies. The cornea offers an ideal site for pathological LG research due to its transparent nature and inducible LG [3]. Since there are no pre-existing or background vessels to consider, it is exceptionally straightforward and accurate to assess LG in response to a pathological insult, such as inflammation.

The Wnt system is known to be involved in multiple processes, such as cell fate determination, stem cell maintenance, and tumor metastasis $[4,5]$. Up to this stage, there is no information on its potential roles in inflammatory LG, which is the focus of this study. Using a repertoire of in vivo inflammatory LG and in vitro cell culture systems and methods, we herein report, for the first time, that monocyte-derived Wnt signaling is involved in inflammatory $L G$ response.

As shown in Figure 1A and 1B, we first set out to investigate whether monocyte-derived Wnt signaling was involved in inflammatory LG using the standard corneal suture placement model [3] and the monocyte conditional knockout mice of Wntless $(W l s)$, a gene encoding a transporter for all Wnt ligands [6]. Targeted deletion of $W l s$ in monocyte lineage was achieved by crossbreeding CsflR-cre and Wls-flox mice. Our results demonstrated a significant reduction of lymphatic coverage area in $W l s^{f l /+} ; c f m s$-icre knockout mice compared with wildtype control littermates. Moreover, real-time PCR data revealed a transcription decrease of vascular endothelial growth factor-C (VEGF-C, a critical mediator of LG [7]) in the corneas of the knockout mice (Figure 1C). In ad- dition, Arginine-1 (Arg-1), a well-characterized gene induced upon macrophage activation, was also down-modulated in the knockout mice (Figure 1C). This gene is a fingerprint of the alternative activated macrophages (M2), which mediate pro-lymphangiogenic response [8]. Taken together, these data indicate that Wnt ligands play a role in regulating inflammatory LG and their secretion by macrophages sustains a high level of VEGF-C within the tissue.

Our further analysis showed that in $W l s$ monocyte conditional knockout mice, there was a significant reduction of Wnt5a in the inflamed corneas, compared with control littermates (Figure 1C). In addition, we performed in situ hybridization analysis on sutured wildtype corneas and confirmed that the presence of Wnt5a transcript in inflamed corneal stroma displays an expression pattern similar to that of F4/80 (a macrophage specific marker) transcript (Supplementary information, Figure S1A). This result indicated macrophage as a major source of Wnt5a in the inflamed cornea and was further corroborated by antibody staining against Wnt5a and F4/80 (Supplementary information, Figure S1B). Wnt5a signals were detected on and around macrophages and lymphatic vessels.

To further investigate the contribution of monocyte-derived Wnt5a to the phenotype, we generated $\mathrm{Wn}$ t5a monocyte conditional knockout mice by crossbreeding CsflR-cre and Wnt5a-flox mice [9]. Interestingly, $W n t 5 a^{A t / f}$; $c$ fms-icre mice also showed reduced LG compared with control $c$ fms-icre littermates with the corneal suture placement model (Figure 1D and 1E). In agreement with the observation in the Wls knockout mice, the transcription levels of VEGF-C and Arg-1 in the corneas were also down-modulated in $W n t 5 a$ knockout mice besides Wnt5a, highlighting a less pro-lymphangiogenic microenvironment (Figure 1F).

We next interrogated whether the phenotype observed in Wnt5 knockout mice was due to impaired homing of macrophages in the inflamed tissues of the transgenic mice. As shown in Figure $1 \mathrm{G}$ and $1 \mathrm{H}$, our results from immunofluorescent microscopic analysis on the macro- 
A

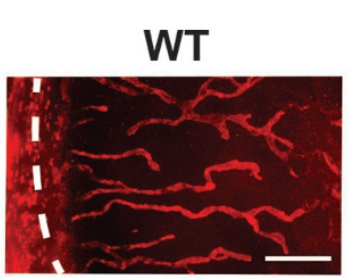

D

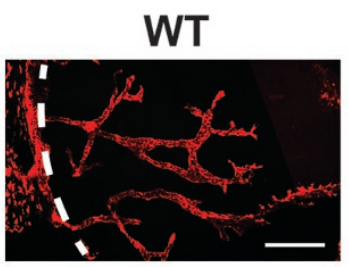

G
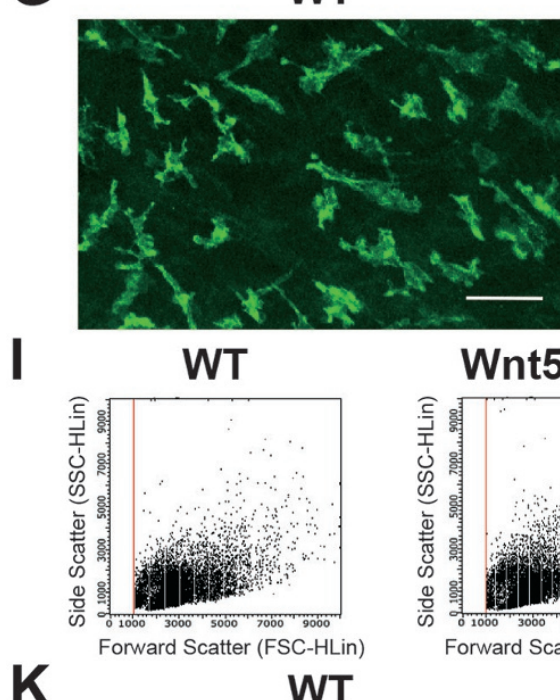

WT
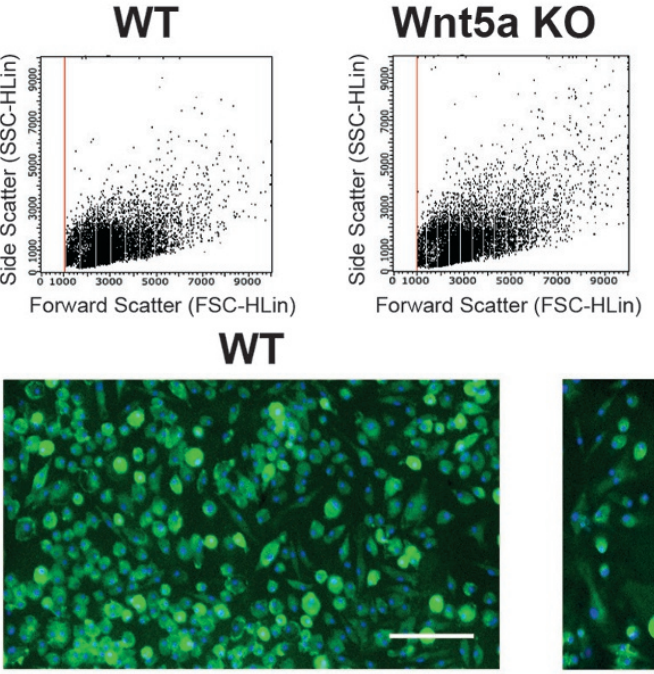

M
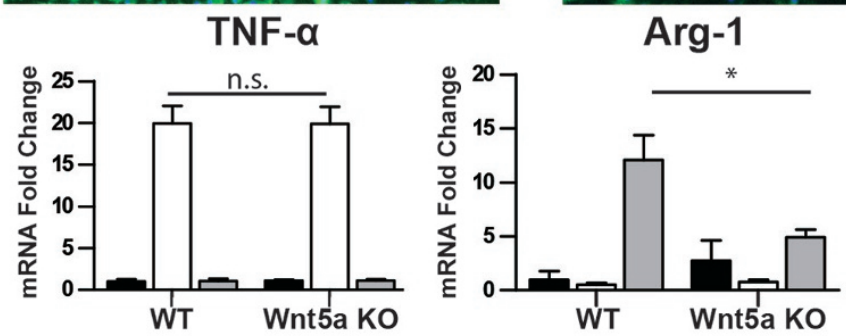

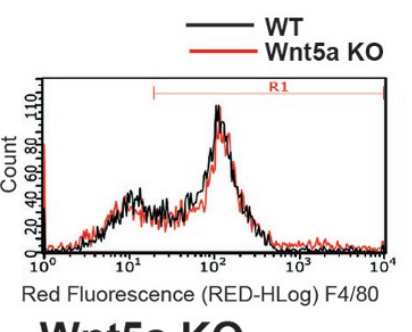

Wnt5a KO

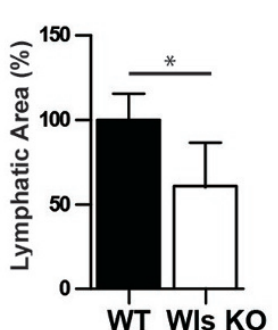

E

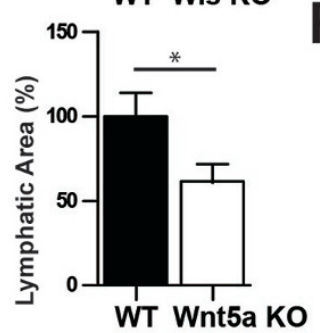

Wnt5a KO
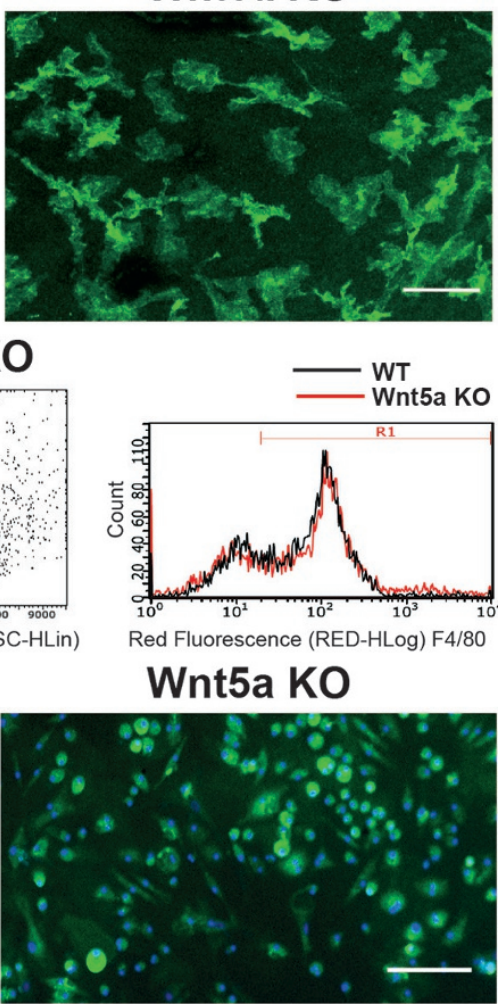

Not Treated

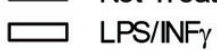

IL-4

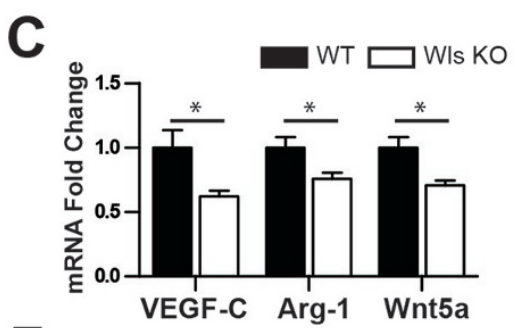

$\mathbf{F}$

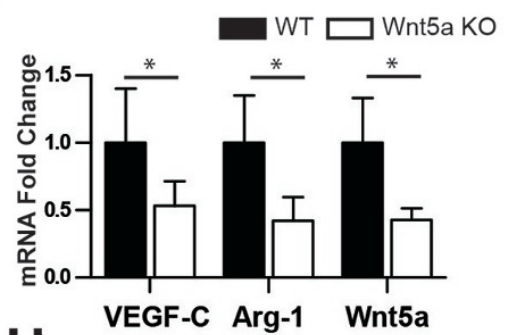

$\mathrm{H}$

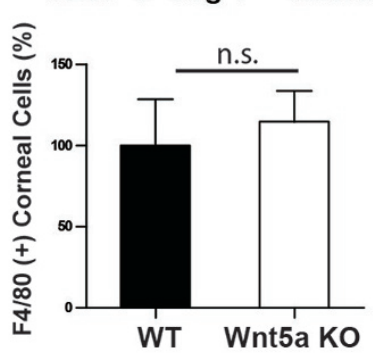

$J$

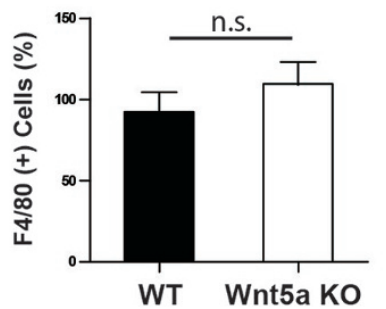

$\mathbf{L}$

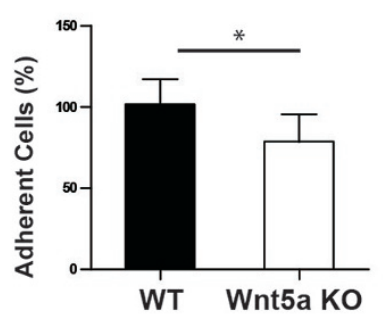

$\mathbf{N}$

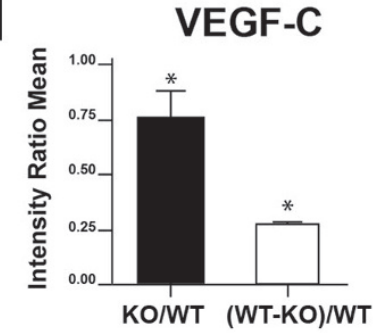

phage-specific marker F4/80 in sutured corneas revealed no significant difference in macrophage infiltration between Wnt5a knockout and control littermates. Similarly, by cytofluorimetric analysis of F4/80 positive cells, no significant difference was observed in the numbers of peritoneal macrophages between the knockout and con- 
trol mice (Figure 1I and 1J). Taken together, these data demonstrate that Wnt5a is dispensable for macrophage homing in peripheral tissues and that the reduced inflammatory LG cannot be ascribed to a reduced number of macrophages in the tissue.

We then investigated in more detail the phenotype of Wnt $5 a$-deficient monocytes and performed in vitro macrophage differentiation assay using bone marrow (BM) monocytes from either Wnt5a monocyte conditional knockout mice or control littermates. As shown in Figure $1 \mathrm{~K}$ and $1 \mathrm{~L}$ and Supplementary information, Figure S1C, 10 days after granulocyte monocyte-colony stimulator factor (GM-CSF) stimulation, we observed a decreased number of $\mathrm{F} 4 / 80^{+}$and $\mathrm{CD} 11 \mathrm{~b}^{+}$cells in Wnt5a knockout mice, as compared with control littermates, indicating a role of Wnt5a in the function of macrophage differentiation.

To further characterize the BM-derived macrophages, we performed in vitro activation assays and analyzed classical (M1) and alternative (M2) responses. We stimulated macrophages with a combination of interferon-gamma (INF- $\gamma$ ) and lipopolysaccharide (LPS) for the M1 activation and interleukin-4 (IL-4) for the M2 activation. Results from our studies showed no difference in the upregulation of TNF- $\alpha$ production in the INF- $\gamma / \mathrm{LPS}$ stimulated macrophages between the Wnt5 knockout and control mice. In contrast, the Arg-1 upregulation upon IL-4 stimulation was impaired in the Wnt5a knockout macrophages (Figure 1M). This last datum suggests that Wnt5a plays a role in skewing macrophages toward the M2 phenotype. Moreover, the down-modulation of Arg1 is in agreement with our previous results from the inflamed corneas, as shown in Figure 1F.
To further corroborate the defective M2 activation of Wnt $5 a$ knockout macrophages, we examined the amount of VEGF-C protein secreted by BM-derived macrophages upon IL-4 stimulation. By arraying the cytokines present in the conditional medium, we determined that the level of VEGF-C was significantly decreased in $\mathrm{Wn}$ t5a knockout macrophages (Figure 1N).

In summary, this study provides the first evidence that Wnt5a is directly involved in the pathological response of inflammatory LG. Moreover, Wnt5a acts through the monocyte-derived cells and regulates VEGF-C production and macrophage phenotype. The nature of this regulation is in contrast to how monocyte-derived Wnt5a regulates blood capillaries in developing retinas where Wnt5a acts via the Flt-1 pathway [10]. This discrepancy is not surprising since Wnt signaling often presents distinct outcomes depending on cell or tissue type and developmental versus pathological stage [11]. Recently, a study on Wnt5a conventional (non-conditional) knockout mice showed reduced number but increased size of dermal lymphatic capillaries at embryonic day 18.5 [12], which indicates a developmental defect resulting from cumulative role of Wnt5a in all cell types, known and yet-to-be determined. In contrast, we observed reduced number but not increased size of lymphatic vessels in this inflammation study on adult mice taking advantage of the cell type-specific conditional knockout mice. Our work reveals and advocates a critical role for monocyte-derived Wnt5a in regulating inflammatory LG. Further investigation on this pathological phenomenon holds great promise for developing novel cell- and/or molecule-based therapies for the widely occurring LG diseases in the body.

Figure 1 Monocyte-derived Wnt5a regulates inflammatory lymphangiogenesis. (A) Representative images of immunofluorescent microscopic analysis showing significantly reduced lymphatic vessels (LYVE- $1^{+}$, red) in the inflamed cornea of Wls knockout (WIs${ }^{\mathrm{fl} / \mathrm{*}}$; $\mathrm{cfms}$-icre, KO) mice compared with wild-type (WT) control littermates. White dashed line: demarcation between the cornea and conjunctiva. Scale bar: $200 \mu \mathrm{m}$. (B) Summarized data showing the significant difference in lymphatic invasion area between the two groups. (C) qPCR analysis showing reduced gene expression levels of VEGF-C, Arg-1 and Wnt5a in the inflamed corneas of WIs KO mice compared with WT. (D) Representative images of immunofluorescent microscopic analysis showing significantly reduced lymphatic vessels (LYVE-1 $1^{+}$, red) in the inflamed cornea of Wnt5a KO (Wnt5a $a^{\mathrm{f} / f /} ;$ cfms-icre) mice compared with WT control littermates. White dashed line: demarcation between the cornea and conjunctiva. Scale bar: $200 \mu \mathrm{m}$. (E) Summarized data showing the significant difference in lymphatic invasion area between the two groups. (F) qPCR analysis showing reduced gene expression levels of VEGF-C, Arg-1 and Wnt5a in the inflamed corneas of Wnt5a KO mice compared with WT. (G) Representative confocal images of immunofluorescent microscopic analysis showing $\mathrm{F} 4 / 80^{+}$macrophage (green) infiltration in the inflamed corneas of Wnt5a KO and WT mice. No significant difference was identified. Scale bar: $50 \mu \mathrm{m}$. (H) Quantitative data from repetitive experiments. NS: not significant. (I, J) Flow cytometry analysis of peritoneal cells showing no significant difference in the numbers of $\mathrm{F} 4 / 80^{+}$macrophages between Wnt5a KO and WT mice. (K) Representative images of immunocytofluorescent analysis on F4/80 (green) cells 10 days after bone marrow-differentiated macrophage culture with GM-CSF. Fewer cells were observed from Wnt5a KO mice compared with WT. Blue: DAPI nuclear staining. Scale bar: $100 \mu \mathrm{m}$. (L) Summarized data showing the difference in cell coverage area. (M) qPCR data showing differential gene expression of TNF- $\alpha$ and Arg-1 in bone marrow-derived macrophages stimulated overnight with INF- $\gamma$ plus LPS or IL-4, respectively. (N) Signal intensity densitometry analysis from the mouse antibody array showing reduced VEGF-C expression level in the supernatant of bone marrow-derived macrophages stimulated overnight with IL-4. ${ }^{*} P<0.05$. 


\section{Acknowledgments}

This work is supported in part by grants from the National Institutes of Health and the University of California at Berkeley (LC). We acknowledge the following people at University of California at Berkeley for technical assistance: (1) from LC Lab at the Center for Eye Disease and Development, Program in Vision Science, and School of Optometry: Guangyu Li, Gyeong Jin Kang, Eric Huang, Valerie Su, Ying Wen and Narae Lee; and (2) from the Department of Molecular and Cell Biology: Prof John Ngai. We would also like to thank Core Translational Module at Program in Vision Science and School of Optometry at University of California at Berkeley for statistical assistance.

Roberto Sessa ${ }^{1,2}$, Don Yuen ${ }^{1,2}$, Stephanie Wan ${ }^{1,2}$, Michael Rosner ${ }^{1,2}$, Preethi Padmanaban ${ }^{1,2}$, Shaokui Ge ${ }^{2}$, April Smith ${ }^{3}$, Russell Fletcher ${ }^{4}$, Ariane Baudhuin-Kessel ${ }^{4}$, Terry P Yamaguchi ${ }^{5}$, Richard A Lang ${ }^{3}$, Lu Chen ${ }^{1,2}$

${ }^{I}$ Vision Science Graduate Group, University of California, Berkeley, CA, USA; ${ }^{2}$ Center for Eye Disease and Development, Program in Vision Science, and School of Optometry, University of California, Berkeley, CA,
USA; ${ }^{3}$ Visual Systems Group, Cincinnati Children's Hospital Medical Center, Cincinnati, OH, USA; ${ }^{4}$ Department of Molecular and Cell Biology, University of California, Berkeley, CA, USA; ${ }^{5}$ Center for Cancer Research, National Institutes of Health, Frederick, MD, USA

Correspondence: Lu Chen

Tel: +1-510-642-5076

E-mail: chenlu@berkeley.edu

\section{References}

$1 \quad$ Alitalo K. Nat Med 2011; 17:1371-1380.

2 Cueni LN, Detmar M. Lymphat Res Biol 2008; 6:109-122.

3 Chen L. Lymphology 2009; 42:66-76.

4 Nusse R. Cell Res 2005; 15:28-32.

5 Clevers H, Loh KM, Nusse R. Science 2014; 346:1248012.

6 Carpenter AC, Rao S, Wells JM, et al. Genesis 2010; 48:554-558.

7 Cursiefen C, Chen L, Borges LP, et al. J Clin Invest 2004; 113:10401050.

8 Watari K, Shibata T, Kawahara A, et al. PLoS One 2014; 9:e99568.

9 Yun K, Ajima R, Sharma N, et al. Hum Mol Genet 2014; 23:68076814.

10 Stefater JA 3rd, Lewkowich I, Rao S, et al. Nature 2011; 474:511-515.

11 van Amerongen R, Fuerer C, Mizutani M, et al. Dev Biol 2012; 369:101-114.

12 Buttler K, Becker J, Pukrop T, et al. Dev Biol 2013; 381:365-376.

(Supplementary information is linked to the online version of the paper on the Cell Research website.) 